\title{
Dynamic coordination with individual learning
}

\author{
Dasgupta, Amil ; Steiner, Jakub ; Stewart, Colin
}

\begin{abstract}
We study coordination in dynamic global games with private learning. Players choose whether and when to invest irreversibly in a project whose success depends on its quality and the timing of investment. Players gradually learn about project quality. We identify conditions on temporal incentives under which, in sufficiently long games, players coordinate on investing whenever doing so is not dominated. Roughly speaking, this outcome occurs whenever players' payoffs are sufficiently tolerant of non-simultaneous coordination. We also identify conditions under which players coordinate on the risk-dominant action. We provide foundations for these results in terms of higher order beliefs.
\end{abstract}

DOI: https://doi.org/10.1016/j.geb.2011.07.005

Other titles: Dynamic coordination with private learning

Posted at the Zurich Open Repository and Archive, University of Zurich

ZORA URL: https://doi.org/10.5167/uzh-174743

Journal Article

Accepted Version

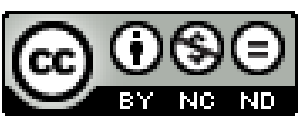

The following work is licensed under a Creative Commons: Attribution-NonCommercial-NoDerivatives 4.0 International (CC BY-NC-ND 4.0) License.

Originally published at:

Dasgupta, Amil; Steiner, Jakub; Stewart, Colin (2012). Dynamic coordination with individual learning. Games and Economic Behavior, 74(1):83-101.

DOI: https://doi.org/10.1016/j.geb.2011.07.005 


\title{
Dynamic Coordination with Individual Learning*
}

\author{
Amil Dasgupta ${ }^{\dagger}$ \\ LSE
}

\author{
Jakub Steiner \\ Northwestern University
}

September 27, 2010

\author{
Colin Stewart ${ }^{\S}$ \\ University of Toronto
}

\begin{abstract}
We study coordination in dynamic global games with private learning. Players choose whether and when to invest irreversibly in a project whose success depends on its quality and the timing of investment. Players gradually learn about project quality. We identify conditions on temporal incentives under which, in sufficiently long games, players coordinate on investing whenever doing so is not dominated. Roughly speaking, this outcome occurs whenever players' payoffs are sufficiently tolerant of non-simultaneous coordination. We also identify conditions under which players coordinate on the risk-dominant action. We provide foundations for these results in terms of higher order beliefs.
\end{abstract}

${ }^{*}$ We thank Martin Cripps, Jeff Ely, Philippe Jehiel, John Moore, Stephen Morris, Emre Ozdenoren, Alessandro Pavan, Eran Shmaya, Ian Stewart, Muhamet Yildiz, and several anonymous referees for helpful comments. We are also grateful for comments from seminar participants at Bonn, Edinburgh, LSE, UCL, the 2007 Workshop on Global Games at Stony Brook, the ESEM 2007 in Budapest, Games 2008 in Evanston, and the ASSA 2008 in New Orleans.

$\dagger$ a.dasgupta@lse.ac.uk

¡j-steiner@kellogg.northwestern.edu

$\S$ colin.stewart@utoronto.ca 


\section{Introduction}

Coordination problems arise in a wide variety of economic situations. A typical example is of a setting where the successful implementation of a risky investment project depends on whether enough agents invest. Strategic uncertainty is an important factor determining on which action the players coordinate. When players are uncertain about other players' actions, they may favor the safe action — not to invest - as opposed to the risky action of investing.

The global games framework (Carlsson and van Damme [3], Morris and Shin [15]) has often been used to model strategic uncertainty in coordination problems. In these games, uncertainty about other players' actions stems from asymmetric information: even if a player is herself optimistic about the project, she may believe that other players could be skeptical and hence may abstain from investment.

The canonical global game features a static framework in which actions are simultaneous and the information of each player does not evolve. In this paper, we focus on dynamic coordination problems in which players may act at different times while they privately learn about the project. How do such dynamic elements impact the nature of strategic uncertainty? In these enriched settings, strategic uncertainty hinges not only on players' beliefs about their opponents' current beliefs, but also about their opponents' beliefs at other stages of the learning process.

In this paper, we characterize rationalizable behavior in a large class of dynamic coordination games. We allow payoffs to depend on the timing of each player's investment choice in a general way. This enables us to examine the nature of strategic uncertainty in a rich variety of circumstances. We introduce a generalized higher order beliefs framework to unify the analysis of this class of problems. Using this framework, we show that small differences in the way payoffs depend on the timing of actions can have radical consequences for behavior. This suggests that caution is warranted when drawing policy implications from the static analysis of fundamentally dynamic coordination problems.

To illustrate the importance of dynamic considerations for strategic uncertainty, consider two extreme variations of a specific coordination problem. Two players decide whether to invest in a project while receiving a sequence of noisy private signals about the state of the project. The players privately learn the true state asymptotically. The 
project succeeds if the state is good and both players invest. The two variations differ in how success depends on the timing of players' investment. In the first variation, the timing of investment is entirely inflexible - for success, players must both invest in a specific period. In the other, the timing of investment is entirely flexible - the precise time at which players invest has no impact on the project's outcome.

The game with inflexible timing is effectively a static global game. Consequently, in the limit as the noise in players' signals vanishes, we know from the global games literature that players coordinate on the risk-dominant action.

Now consider, instead, the game with flexible timing. For tractability, we assume that exactly one of the actions, say investment, is irreversible. ${ }^{1}$ Until they commit to it, players choose in each period whether to invest. The project succeeds if both players invest, not necessarily in the same period. Players do not observe each other's actions (in Section 8 below, we show how to reinterpret this information structure in terms of social learning). The choice between early and late investment is driven by a trade-off: the investment opportunity shrinks over time, but late investors have more accurate private information.

We show that whenever the state is good, the players are very likely to coordinate on the irreversible action at the beginning of the process, i.e., both invest in the first round, as long as they each acquire sufficiently precise information.

If the risk-dominant and irreversible actions do not coincide, the outcomes of the flexible and inflexible timing games may be significantly different. To understand why this is the case, suppose that investment is not risk-dominant in the inflexible variant of the game. This means that a necessary condition for each player to invest is that she assigns probability greater than $1 / 2$ to the success of the project (conditional on her own investment).

The problem that precludes coordination on investing in the inflexible game has its roots in the nature of players' second-order beliefs: for any $p$, a player who assigns probability $p$ to the state being good assigns probability $1 / 2$ to her opponent being less confident than herself about the state. Therefore, there does not exist a sufficient confidence level $p$ above which players invest; if such a $p$ existed, then a player with confidence level $p$ strictly prefers not to invest, for even if she is optimistic about the

\footnotetext{
${ }^{1}$ This assumption is concordant with a large literature on irreversible investment under uncertainty, see, e.g., Dixit and Pindyck [7].
} 
state, she assigns probability $1 / 2$ to the other player not investing.

In contrast, in the flexible game, the problem with second-order beliefs disappears. In this case, each player asks a much less demanding question about her opponent's belief and behavior, namely, whether her opponent attains the requisite belief at some point over the course of the game. As a result, there is a sufficient confidence level $p$ above which players invest. Suppose a type assigns probability $p$ to the state being good. If the state is indeed good then it is almost inevitable that the opponent shares this belief at least once during a sufficiently long learning process. Hence if a type assigns a high probability to the state being good, she also assigns a high (albeit slightly smaller) probability to the opponent investing during the game. We show later in the paper that, although a trace of strategic uncertainty remains in the flexible game, it does not undermine coordination on investing.

The flexible and inflexible games represent extremes of the range of games we consider. Our higher order beliefs framework may be applied to games with more realistic preferences over the timing of actions taken by one's opponent. Section 3 introduces two such games. In the first of these games, each player prefers not to invest before the other player invests: there are small losses for being (exclusively) the first to invest. In the second, each player prefers to invest one period before the other player invests: there is a small reward for being (exclusively) the first to invest. We show that players coordinate on the risk-dominant action in the first game. In contrast, when learning is sufficiently fast, players coordinate on the irreversible action in the second game. Thus small changes in timing preferences can have dramatic effects on outcomes.

The analysis of higher order beliefs has proved to be useful in explaining strategic behavior in static coordination games. As discussed above, in order to invest, a first-order belief that the project is sound is not sufficient. Players must also have a sufficient second-order belief that other players are sufficiently sure about the soundness of the project, a sufficient third-order belief, etc. In fact, players coordinate on investment only if they achieve a sufficient degree of approximate common knowledge that the project is sound. However, for the information structure of the canonical global game, approximate common knowledge of the project's quality is never attained. Thus strategic uncertainty may preclude coordination on investment in the inflexible game. Building on the standard notion of approximate common knowledge due to Mon- 
derer and Samet [13], we construct a generalization that is suitable for characterizing behavior in dynamic coordination games. This allows us to unify the analysis of a wide class of dynamic games and to highlight the role of higher order beliefs. Our generalization lies in allowing the required beliefs to be attained at different times. For each game we study, we utilize a particular version of the generalized belief operators that reflects preferences over the timing of investment in that game.

\section{$1.1 \quad$ Literature Review}

The present paper bears a general connection to models of information dynamics in multi-stage global games. One strand of the literature (e.g., Chamley [4], Angeletos, Hellwig and Pavan [2]) focuses on the robustness of equilibrium uniqueness in global games when learning is based on endogenously generated public signals. This paper belongs to the other strand that focuses on settings with pure private information. This strand originated in the work of Dasgupta [6] who documents the effect of private learning on coordination in two-stage global games. ${ }^{2}$ We explain behavior in a related dynamic framework in terms of higher order beliefs.

Our generalization of approximate common knowledge builds on the work of Monderer and Samet [13] and Morris and Shin [14]. The analysis is also related to the work of Cripps, Ely, Mailath, and Samuelson [5] (henceforth CEMS), who delineate general conditions under which agents asymptotically attain approximate common knowledge via private learning. The analysis of CEMS is relevant in situations which can be divided into two distinct phases: agents learn privately in the first phase, and attempt to coordinate simultaneously in the second. We study situations in which those two phases are merged together: players attempt to coordinate while they privately learn about payoffs. Both papers study whether private learning leads to approximate common knowledge; however, the relevant form of approximate common knowledge depends on the intertemporal incentives in the game. CEMS study standard common beliefs as defined in Monderer and Samet [13], while we study a more general form of common beliefs suitable when coordination need not be simultaneous. The two concepts turn out to have very different properties. In our model, private learning fails to deliver common knowledge in the standard sense, but succeeds in delivering a relaxed form

\footnotetext{
${ }^{2}$ Another example of a dynamic global game with private learning can be found in Heidhues and Melissas [11].
} 
of common beliefs. This explains why coordination failure arises in the game with inflexible timing but does not arise in the game with flexible timing. ${ }^{3}$

Gale [9] provides an elegant analysis of the extent of inefficient delay in dynamic coordination games with complete information. He shows that inefficient delay can be eliminated when the period length becomes very small. Hörner [12] and Xue [17] similarly obtain efficient coordination in incomplete information games with observable irreversible actions. While in some settings our main result implies delay-free coordination on investing in the limit as private signals become accurate, and thus bears a resemblance to Gale's, the forces driving the results are very different. Gale's result builds on backward induction based on the observability of past actions in a game of perfect information, while we consider an asymmetric information setting in which players do not observe each others' actions. When the efficient action is irreversible, Gale's observability assumption favors efficient coordination by eliminating strategic uncertainty once a player has chosen the irreversible action. Our results indicate that coordination on the irreversible action does not require observability per se; it may arise even in games with large numbers of players where perfect observation of actions is not feasible. In Section 8 below, we describe how to reinterpret our model as one of noisy social learning in a large population.

\section{Example}

In order to highlight the key ideas of the general case, we devote this section to an analysis of the flexible and inflexible games. Our aim at this point is to cover most of the insights without getting too deeply into technicalities. Thus we postpone discussion of the solution concept (which is rationalizability). We also postpone the introduction of our general higher order beliefs framework, and the description of more realistic (but also more complex) applications. The two main results of this section have more general counterparts in Propositions 3 and 5.

We start by describing parts of the setup that are common to both games. Two players participate in a joint investment game $\Gamma_{T}$ with $T$ periods, where $T \in \mathbb{N}$. The

\footnotetext{
${ }^{3}$ Ely [8] informally discusses a notion of common belief relevant for non-simultaneous coordination, but only to contrast it to the standard common belief which is the relevant concept for the problems he considers.
} 
action of player $i$ in period $t \in\{1, \ldots, T\}$ is denoted by $a_{i t} \in\{0,1\}$; we interpret action 1 as "invest", and 0 as "wait". Investment is irreversible. To keep notation simple, players choose an action in each round but action choices become payoff-irrelevant after a player has invested once. Whenever we refer to an action choice, we implicitly condition on the player not having invested in an earlier round.

The payoffs in the game depend on the action profile and the value of a fundamental parameter $\theta$. The fundamental $\theta$ is drawn before the first round from an improper uniform distribution on $\Theta=\mathbb{R}$, and remains fixed over all rounds.

The players do not observe $\theta$ directly, but receive private noisy signals of $\theta$ in every round. Specifically, each player $i$ receives a signal $y_{i t}=\theta+\nu_{t} \varepsilon_{i t}$ in round $t$, where the errors $\varepsilon_{i t}$ are standard normal random variables that are independent across players and rounds. The standard errors $\nu_{t}$ are strictly positive for all $t$, and the sequence $\left(\nu_{t}\right)_{t=1}^{\infty}$ is fixed throughout independent of the value of $T$, which we vary. Players do not observe each other's actions. ${ }^{4}$

Players form beliefs about $\theta$ in each period through Bayesian updating. Conditional on signals $\left(y_{i s}\right)_{s=1}^{t}$, player $i$ believes that $\theta$ is distributed as $N\left(x_{i t}, \sigma_{t}^{2}\right)$, where

$$
x_{i t}=\sum_{s=1}^{t} y_{i s} \frac{\sigma_{t}^{2}}{\nu_{s}^{2}}, \text { and } \frac{1}{\sigma_{t}^{2}}=\sum_{s=1}^{t} \frac{1}{\nu_{s}^{2}}
$$

We refer to $x_{i t}$ as the cumulative signal, and to $\sigma_{t}$ as the cumulative standard error. We assume throughout that each player's strategy depends, in each period, only on her cumulative signal, not on the full sequence of signals to date.

We assume that players asymptotically privately learn the true fundamental, that is, $\lim _{t \rightarrow \infty} \sigma_{t}=0$. Note that, since each $\nu_{t}$ is strictly positive, each cumulative standard error $\sigma_{t}$ is also strictly positive. Thus even though players learn the true fundamental in the limit over all periods, some uncertainty remains in each round. ${ }^{5}$

The outcome of the project is binary: success or failure. Which outcome occurs is determined at the end of the game by the fundamental $\theta$ and the actions of the players. For $\theta \leq 0$ or $\theta \geq 1$, the project fails or succeeds, respectively, regardless of

\footnotetext{
${ }^{4}$ However, as we demonstrate in Section 8, under certain circumstances, the signals $y_{i t}$ can be thought to arise from noisy social learning based on the observation of past actions.

${ }^{5}$ Note that $\nu_{t}$ is not required to converge to 0 . For instance, players asymptotically learn $\theta$ if $\nu_{t}$ is constant.
} 
the players' actions; we call these intervals the dominance regions. For $\theta \in(0,1)$, the project succeeds if and only if both players invest by round $T$, possibly at different times.

We compare two versions of the game: the inflexible and the flexible one. In the inflexible game, the players choose an action only at round $T$. In the flexible game the players can (irreversibly) invest in any period $t \in\{1, \ldots, T\}$.

We assume the following properties of the best response correspondences.

Assumption. There exist some $\underline{p}, \bar{p} \in(0,1)$ such that,

- (necessary condition for investing) in the inflexible game, each player prefers to invest only if she assigns probability greater than $\underline{p}$ to the success of the project (provided she invests).

- (sufficient condition for investing) in the flexible game, each player prefers to invests in period $t$ whenever she assigns probability greater than $\bar{p}$ to the success of the project (provided she invests).

One example in which the assumption holds is given by the following payoffs: never investing yields a payoff of 0 ; investing in round $t$ yields $\delta^{t} b$ (respectively $-\delta^{t} c$ ) if the project succeeds (respectively fails). The parameters $b$ and $c$ are both strictly positive, and $\delta \in(0,1)$ captures the rate of "shrinking of the investment opportunity." It is easy to verify that the trade-off between investing early when the investment opportunity is large and waiting for additional information implies the above best-response property in the flexible game with $\bar{p}=\frac{c}{(1-\delta) b+c}$. In the inflexible game, the property holds with $\underline{p}=\frac{c}{b+c} \cdot{ }^{6}$

First consider the inflexible game. The following proposition implies that players coordinate on investment only if they believe that it is the dominant action (i.e. that $\theta>1)$.

Proposition 1. Suppose $\underline{p}>\frac{1}{2}$. There exists $q \in(0,1)$ such that each player invests in the inflexible game only if she q-believes that $\theta>1$.

\footnotetext{
${ }^{6}$ Note that the irreversible action in this example corresponds to the efficient outcome for positive $\theta$. However, efficiency per se plays no role in the analysis. To see this, consider the following modification. In addition to the above payoffs, each player incurs a penalty of $2 b$ whenever her opponent invests. Since this modification does not affect the best response correspondence, rationalizable behavior remains the same while coordination on investing is now Pareto dominated by mutual non-participation.
} 
This result can be, in various forms, found in the extant literature on static global games (see Morris and Shin [15]), and so we only briefly describe the intuition here. Suppose that the two players use symmetric strategies characterized by a threshold cumulative signal $x^{*}$ at time $T$ above which each invests and below which each does not invest. A player who receives exactly the threshold signal must be indifferent between investing and not investing. The threshold type, regardless of the precision of her private signal, suffers from strategic uncertainty. By symmetry, she assigns probability exactly $1 / 2$ to her opponent receiving a signal above $x^{*}$, and hence probability $1 / 2$ to her opponent investing. Since $\underline{p}>1 / 2$, not investing is a strict best response unless the player assigns high enough probability to $\theta>1$.

In contrast to the previous proposition, for sufficiently large $T$, players coordinate on the irreversible action in the flexible game whenever they believe that this action is not dominated (i.e. that $\theta>0$ ).

Proposition 2. For any $q \in(\bar{p}, 1)$ there exists some $\underline{T}$ such that for any $T>\underline{T}$, each player invests in any period $t$ of the flexible game of length $T$ whenever she q-believes at $t$ that $\theta>0$.

Propositions 1 and 2 imply a stark difference between the coordination outcomes across the two games. Consider the limit in which players receive increasingly precise signals in all periods; that is, multiply every cumulative standard error by a common scaling factor $\sigma$ and consider the limit as $\sigma \rightarrow 0$. Consider a particular realization of $\theta \in(0,1)$, where neither action is dominant. The values of $q$ in Proposition 1 and $\underline{T}$ in Proposition 2 are uniform across $\sigma$. Thus, in the limit, in the flexible game, both players irreversibly invest immediately in round 1. In the inflexible game, players do not invest.

As discussed in the introduction, the stark difference in behavior across the two games is a caused by a subtle difference in the nature of strategic uncertainty. In the inflexible game, a player who believes that the project is sound may doubt whether her opponent shares this belief. In the flexible game, this problem with second-order uncertainty is mitigated. In the remainder of this section we discuss the lack of strategic uncertainty in the flexible game in more detail.

Before proving Proposition 2, we briefly outline some notation and formulate a core lemma that establishes the lack of strategic uncertainty in the flexible game. We then 
use a contagion argument to show that the lack of strategic uncertainty implies the result.

For any $q \in(0,1)$ let $E_{i t}^{q}\left(\theta^{*}\right)$ denote the event that player $i q$-believes $\theta>\theta^{*}$ in period $t$; that is, that $x_{i t} \geq \theta^{*}+\sigma_{t} \Phi^{-1}(q)$, where $\Phi$ is the standard normal distribution function. Denote by

$$
l_{T}^{q}\left(\theta, \theta^{*}\right)=\operatorname{Pr}\left(\bigcup_{t=1}^{T} E_{i t}^{q}\left(\theta^{*}\right) \mid \theta\right)
$$

the probability that, when the realized fundamental is $\theta$, the player $q$-believes in at least one round up to $T$ that the fundamental exceeds $\theta^{*}$. One can show that the function $l_{T}^{q}\left(\theta, \theta^{*}\right)$ is continuous and increasing in $\theta$.

Let $l^{q}\left(\theta, \theta^{*}\right)=\lim _{T \rightarrow \infty} l_{T}^{q}\left(\theta, \theta^{*}\right)$. This limit exists because $l_{T}^{q}\left(\theta, \theta^{*}\right)$ is non-decreasing in $T$ and bounded from above by 1 . The following lemma is crucial for our claim that strategic uncertainty is mitigated in the flexible game.

Lemma 1. For each $q \in(0,1)$ and each $\theta^{*}, l^{q}\left(\theta^{*}, \theta^{*}\right)=1$ and $l^{q}\left(\theta, \theta^{*}\right)$ is continuous in $\theta$ at $\theta^{*}$.

When $\theta^{*}=0$ the lemma implies that, as $T \rightarrow \infty$, the probability that each player $q$-believes that the fundamental is positive at least once during the game converges to 1 uniformly across all $\theta \geq 0$. Thus, in a relaxed sense, the players do not suffer from second-order uncertainty: if a player believes that the fundamental is positive then she also believes that her opponent shares this belief at least once over the course of a sufficiently long game. Compare this to the inflexible setup, in which a player may believe that the fundamental is good but still doubt whether the opponent shares this belief. At a formal level, the relevant object in the inflexible game that corresponds to $l_{T}^{q}\left(\theta^{*}, \theta^{*}\right)$ is $\operatorname{Pr}\left(E_{i T}^{q}\left(\theta^{*}\right) \mid \theta^{*}\right)$. Unlike $l_{T}^{q}\left(\theta^{*}, \theta^{*}\right)$, this expression does not tend to 1 as $T$ goes to infinity.

The proof of the lemma is based on the assumption that players asymptotically learn the fundamental. Conditional on the fundamental being equal to $\theta^{*}$, the probability that a player does not $q$-believe $\theta>\theta^{*}$ is $q$ in each round, but with the complication that the posterior probabilities $\operatorname{Pr}\left(\theta>\theta^{*} \mid x_{i t}\right)$ are correlated across periods. We show, roughly, that beliefs across sufficiently distant rounds $t$ and $t^{\prime}$ are approximately independent. The intuition is that if the amount of information that a player receives between $t$ and $t^{\prime}$ is large relative to what she knew at $t$, then the information at $t$ 
has only a negligible impact at $t^{\prime}$. For long games, we can choose a long subsequence of rounds such that all rounds in the subsequence are sufficiently distant. Hence the probability that the player fails to $q$-believe $\theta>\theta^{*}$ in all of these rounds approaches 0 as the length of the subsequence grows large. ${ }^{7}$

Proof of Proposition 2. Fix $q \in(\bar{p}, 1)$ throughout. Let $S(\underline{\theta})$ denote the following statement:

There exists some $\underline{T}$ such that for every $T>\underline{T}$, each player in the game

$\Gamma_{T}$ invests at time $t$ if she $q$-believes at $t$ that $\theta \geq \underline{\theta}$.

The statement clearly holds for $\underline{\theta} \geq 1$. We establish the following contagion step. If for some $\theta^{\prime} \geq 0$, the statement $S(\underline{\theta})$ holds for all $\underline{\theta}>\theta^{\prime}$, then $S\left(\theta^{\prime}\right)$ holds, and, moreover, if $\theta^{\prime}>0$, then there exists some $\varepsilon>0$ such that the statement $S(\underline{\theta})$ holds for all $\underline{\theta}>\theta^{\prime}-\varepsilon$. Let $\theta^{\prime \prime}$ denote the infimum of those $\theta$ for which $S(\theta)$ holds. We must have $\theta^{\prime \prime}=0$, for otherwise taking $\theta^{\prime}=\theta^{\prime \prime}$ contradicts the contagion step. It follows that $S(\underline{\theta})$ holds for all $\underline{\theta}>0$ and hence $S(0)$ holds, as needed.

It remains to establish the contagion step. Suppose that, for some $\theta^{\prime} \geq 0, S(\underline{\theta})$ holds for all $\underline{\theta}>\theta^{\prime}$. Fix some $r \in(\bar{p} / q, 1)$. Lemma 1 implies that there exists $\underline{\theta}>\theta^{\prime}$ such that

$$
l^{q}\left(\theta^{\prime}, \underline{\theta}\right)>r .
$$

Hence there exists some $T^{\prime}$ such that

$$
l_{T^{\prime}}^{q}\left(\theta^{\prime}, \underline{\theta}\right)>r .
$$

Since the function $l_{T^{\prime}}^{q}\left(\theta, \theta^{*}\right)$ is continuous in $\theta$, there exists some $\varepsilon>0$ such that

$$
l_{T^{\prime}}^{q}\left(\theta^{\prime}-\varepsilon, \underline{\theta}\right)>r .
$$

Since $l_{T}^{q}\left(\theta, \theta^{*}\right)$ is increasing in $T$ and $\theta$, we have

$$
l_{T}^{q}(\theta, \underline{\theta})>r
$$

for all $T>T^{\prime}$ and $\theta>\theta^{\prime}-\varepsilon$.

\footnotetext{
${ }^{7}$ We are grateful to an anonymous referee for providing a particulary instructive proof formalizing this intuition.
} 
Suppose the statement $S(\underline{\theta})$ holds with $\underline{T}=T^{\prime \prime}$. Consider a game $\Gamma_{T}$ with $T>$ $\max \left\{T^{\prime \prime}, T^{\prime}\right\}$. Suppose, for some $t$, player $i q$-believes at $t$ that $\theta>\max \left\{0, \theta^{\prime}-\varepsilon\right\}$. Since $T>T^{\prime}$, the inequality $\theta>\theta^{\prime}-\varepsilon$ implies that $l_{T}^{q}(\theta, \underline{\theta})>r$. Since $T>T^{\prime \prime}$, by hypothesis, player $-i$ invests in any period at which she $q$-believes that $\theta>\underline{\theta}$. Thus, conditional on $\theta>\theta^{*}-\varepsilon$, the probability that player $-i$ invests exceeds $r$. Therefore, at $t$, player $i$ attaches probability at least $r q$ to the event that the project succeeds. Since $r q>\bar{p}$, this implies that investing is the best response for player $i$ at $t$.

\section{Additional Games}

Before developing a general higher order beliefs framework for solving a large class of dynamic coordination games, we outline two additional games exhibiting a mixture of features from the inflexible and flexible games. In these additional games, although both players do not have to invest at the same time for the project to succeed, the relative timing of their investment affects their payoffs. In the first of these games, when $\theta \in(0,1)$, each player prefers not to invest before the other player invests: there are small losses for being (exclusively) the first to invest. In the second, each player prefers to invest one period before the other player invests: there is a small reward for being (exclusively) the first to invest.

The players, action sets, and information structures remain the same and investment continues to be irreversible as in Section 2; only the payoffs differ. In both games players receive flow payoffs that depend on whether the other player has invested. The rules for success of the project are the same as in Section 2 but success is determined during the coordination process instead of at the end.

Recalling that investing is irreversible, let $\hat{a}_{i t}=\max \left\{a_{i 1}, \ldots, a_{i t}\right\}$ indicate whether player $i$ has invested up to $t$ ( $\hat{a}_{i t}$ differs from $a_{i t}$ only for convenience; we assume that once a player has committed to investing, her subsequent actions are payoff-irrelevant).

Game 1 (in which neither player wants to invest before the other). Let $s_{t}$ be a success indicator at round $t$, defined by

$$
s_{t}= \begin{cases}1 & \text { if } \hat{a}_{i t}=\hat{a}_{-i t}=1 \text { and } \theta>0, \text { or if } \theta \geq 1, \\ 0 & \text { otherwise. }\end{cases}
$$


One may think of $s_{t}$ as indicating whether the project has succeeded if we take into account only actions up to $t$. Each player $i$ maximizes the discounted sum

$$
\frac{1}{1-\delta} \sum_{t=1}^{T} \delta^{t} u_{i t}
$$

of flow payoffs $u_{i t}$, where

$$
u_{i t}= \begin{cases}0 & \text { if } \hat{a}_{i t}=0 \\ b & \text { if } \hat{a}_{i t}=1 \text { and } s_{t}=1, \\ -c & \text { if } \hat{a}_{i t}=1 \text { and } s_{t}=0 .\end{cases}
$$

One may readily verify that, in Game 1, investing is a best response for player $i$ at time $t$ only if she $p$-believes at $t$ that the project will succeed at $t$ (if she invests), where $\underline{p}=\frac{c}{b+c}$.

Game 2 (in which each player wants to invest before the other). This game is identical to the previous one except that players begin to receive the payoff $b$ one period before the project succeeds. That is, flow payoffs are defined by

$$
u_{i t}= \begin{cases}0 & \text { if } \hat{a}_{i t}=0, \\ b & \text { if } \hat{a}_{i t}=1, \text { and } s_{t+1}=1, \\ -c & \text { if } \hat{a}_{i t}=1 \text { and } s_{t+1}=0,\end{cases}
$$

where $s_{T+1}$ is defined to be equal to $s_{T}$.

Let $\bar{p}=\frac{\delta b+c}{b+c}$. In Game 2, investing is a best response for player $i$ at time $t$ if she $\bar{p}$-believes at $t$ that (if she invests) the project will succeed at or before time $t+1$.

We show in Section 7 that behavior in Game 1 is the same (for large $T$ and small $\sigma)$ as in the inflexible game of Section 2: players choose the risk-dominant action. In contrast, when learning is sufficiently fast, the small incentive to invest just before the opponent leads to coordination on the irreversible action in Game 2, exactly as in the flexible game (again for large $T$ and small $\sigma$ ). 


\section{General Game and the Solution Concept}

Throughout the remainder of the paper, we retain the same set of players, and same information and action structure as described in Section 2, but allow for more general payoffs. We restrict attention to symmetric, common value games in which payoffs depend only on $\theta$ and chosen actions, and not on players' signals. We focus on asymptotic results characterizing behavior in games with many rounds and small noise. We denote by $\Gamma=\left(\Gamma_{T}(\sigma)\right)_{T, \sigma}$ a class of games with varying noise-scaling factor $\sigma>0$ and number of rounds $T \in \mathbb{N}$. The payoff functions may vary across $T$ but not across $\sigma$.

We refer to each pair $(i, t)$ as an agent. The type of agent $(i, t)$, denoted by $x_{i t} \in$ $X_{i t}=\mathbb{R}$, consists of player $i$ 's cumulative signal at time $t$. A strategy for player $i$ is a collection $s_{i}=\left\{s_{i t}\right\}_{t=1}^{T}$ of measurable functions $s_{i t}: X_{i t} \longrightarrow\{0,1\}$. Let $U_{i}\left(a, s_{i}, s_{-i} \mid x_{i t}\right)$ denote the expected payoff for type $x_{i t}$ choosing action $a$ conditional on player $i$ having chosen the reversible action 0 in every period $1, \ldots, t-1$ and following strategy $s_{i}$ in every period $t+1, \ldots, T$. Letting $\mathcal{A}$ denote the set of strategies for each player, define the $\left(\right.$ interim $\left.^{8}\right)$ best response correspondence $\mathrm{BR}(\cdot)$ over subsets $S \subseteq \mathcal{A}$ by

$$
\operatorname{BR}(S)=\left\{s_{i} \mid \forall x_{i t} \exists s_{-i} \in S \text { such that } s_{i t}\left(x_{i t}\right) \in \underset{a \in\{0,1\}}{\operatorname{argmax}} U_{i}\left(a, s_{i}, s_{-i} \mid x_{i t}\right)\right\} .
$$

Note that, since we restrict our attention to symmetric games, we do not distinguish between the best response correspondences of the two players. The set of (interim) rationalizable strategies in game $\Gamma_{T}(\sigma)$ is defined to be $\bigcap_{n=1}^{\infty} \operatorname{BR}^{n}(\mathcal{A})$. We say that action $a$ is rationalizable for $x_{i t}$ if there exists a rationalizable strategy $s_{i}$ such that $s_{i}\left(x_{i t}\right)=a$.

For simplicity, the definition of strategies above implicitly makes two related assumptions: players do not use mixed strategies and their strategies depend only on their cumulative signal, not on the full history of signals they have received. Neither of these assumptions is important for our results. For the former, since each type has only two actions, any action that is never a best response for some type against a set of pure strategies cannot be a best response against a mixture of those pure strate-

\footnotetext{
8 "Interim" refers to the feature that every type of every agent chooses an optimal action given its beliefs about types of other agents (as opposed, for example, to every player choosing an optimal strategy at the ex ante stage). Since we consider only interim best responses, we omit the term "interim" throughout.
} 
gies. Therefore, the set of rationalizable mixed strategies would consist precisely of all distributions over the set of rationalizable pure strategies (as defined above). Similarly, conditioning strategies on full histories of signals is payoff-equivalent to some mixed strategy that depends only on cumulative signals. For any type with a unique rationalizable action, allowing such strategies would have no effect.

Notions of rationalizability in dynamic games are typically more complicated than the one employed here. The main complication is how players revise conjectures about their opponents' strategies that are contradicted by their opponents' actions (see, e.g., Pearce [16]). This issue does not arise in our model because actions are unobservable.

\section{Generalized Higher Order Beliefs}

It is well known that approximate common knowledge plays an important role in determining the outcome of static coordination games. The current section develops a generalization of the notion of approximate common knowledge that is relevant for a large class of dynamic coordination games.

\subsection{Preliminary notation and definitions}

The ordinary state space is $\Omega=\Theta \times\left(\times_{i t} X_{i t}\right)$ (we omit dependence on $T$ from the notation here and elsewhere). Let $\Sigma_{\Omega}$ denote the Borel $\sigma$-algebra on $\Omega$ (endowed with the usual Euclidean topology) and $\Sigma_{\Theta}$ the Borel $\sigma$-algebra on $\Theta$. An element of $\Sigma_{\Omega}$ is an ordinary event. Note that the posterior beliefs of each agent $(i, t)$ over ordinary events are well-defined and continuous in the uniform topology as the type $x_{i t}$ varies.

To conveniently describe strategy profiles, we follow Morris and Shin [14] and introduce compound events. A compound event $F$ is a vector $\left(F_{i t}\right)_{i t}$ of length $2 T$ in which each component $F_{i t}$ is a Borel-measurable subset of the type space $X_{i t}$. We identify each compound event $F=\left(F_{i t}\right)_{i t}$ with the strategy profile in which each agent $(i, t)$ invests if and only if $x_{i t} \in F_{i t}$. Let $\mathcal{S}$ denote the class of all compound events.

For two compound events $F, F^{\prime} \in \mathcal{S}$, we write $F \subseteq F^{\prime}$ if $F_{i t} \subseteq F_{i t}^{\prime}$ for each $(i, t)$. We define binary operations $\wedge$ and $\vee$ by

$$
F \wedge F^{\prime}=\left(F_{i t} \cap F_{i t}^{\prime}\right)_{i t} \quad \text { and } \quad F \vee F^{\prime}=\left(F_{i t} \cup F_{i t}^{\prime}\right)_{i t}
$$


Negation of a compound event is defined as $\neg F=\left(\overline{F_{i t}}\right)_{i t}$, where $\overline{F_{i t}}$ denotes the complement of the set $F_{i t}$. Note that the class of compound events $\mathcal{S}$ is closed under the operations $\wedge, \vee$, and $\neg$. We abuse notation by writing $\emptyset$ for the empty compound event defined as the negation of $\mathbf{X}=\left(X_{i t}\right)_{i t}$.

For each agent $(i, t)$ and $p \in[0,1]$, define the belief operator $B_{i t}^{p}: \Sigma_{\Omega} \longrightarrow 2^{X_{i t}}$ by

$$
B_{i t}^{p}(E)=\left\{x_{i t} \in X_{i t}: \operatorname{Pr}\left(E \mid x_{i t}\right) \geq p\right\}
$$

Thus, $B_{i t}^{p}(E)$ is the set of types of agent $(i, t)$ that $p$-believe the ordinary event $E$.

Let $B^{p}:\left[\Sigma_{\Omega}\right]^{2 T} \longrightarrow \mathcal{S}$ denote the vector of belief operators given by

$$
\left[B^{p}(E)\right]_{i t}=B_{i t}^{p}\left(E_{i t}\right)
$$

where $E=\left(E_{i t}\right)_{i t}$ is a vector of ordinary events $E_{i t} \in \Sigma_{\Omega}$. Thus $B^{p}(E)$ is a strategy profile under which agent $(i, t)$ invests if and only if she $p$-believes $E_{i t}$.

\subsection{Necessary and sufficient conditions for investment}

We now introduce a formalism describing conditions on the timing of one's opponent's investment under which a player prefers to invest. These conditions, describing many varieties of dynamic coordination games, are the primitives of our model. In the next section, we connect these conditions on timing of investment to the belief operators $B_{i t}^{p}$. We develop generalized beliefs that are behaviorally relevant in our class of dynamic games.

Let $U=[1, \infty)$ denote the upper dominance region, and $G=(0, \infty)$ denote the set of "good" fundamentals. Roughly, we assume that each player prefers to invest if she believes either that the fundamental is very good $(\theta \in U)$, or that the fundamental is good and the timing of the opponent's investment satisfies certain conditions.

These conditions make use of operators $O_{i t}^{T}: \mathcal{S} \longrightarrow \Sigma_{\Omega}$ that map strategy profiles to ordinary events. We denote by $O^{T}=\left(O_{i t}^{T}\right)_{i t}$ the vector of these operators. Finally, we denote by $O=\left(O^{T}\right)_{T}$ the sequence of operators $O^{T}$. Note that $O$ is independent of $\sigma$. When there is no risk of confusion, we sometimes omit dependence of $O^{T}$ and $O_{i t}^{T}$ on $T$. Let $p \in[0,1]$.

Definition 1. We say that $(O, p)$ describes sufficient conditions for investment 
in a class of games $\Gamma$ if for any strategy profile $F$, in every game $\Gamma_{T}(\sigma)$, action 1 is the unique best response for type $x_{i t}$ whenever

$$
x_{i t} \in B_{i t}^{p}\left(\left[G \cap O_{i t}^{T}(F)\right] \cup U\right) .
$$

Definition 2. We say that $(O, p)$ describes necessary conditions for investment in a class of games $\Gamma$ if for any strategy profile $F$, in every game $\Gamma_{T}(\sigma)$, action 1 is a best response for type $x_{i t}$ only if

$$
x_{i t} \in B_{i t}^{p}\left(\left[G \cap O_{i t}^{T}(F)\right] \cup U\right)
$$

Since we focus on symmetric games, we assume that $O_{i t}$ is symmetric across the players. To illustrate the definitions, consider the games in Sections 2 and $3 .^{9}$

- In the flexible game of Section $2,(O, p)$ describes sufficient conditions for investment with

$$
O_{i t}(F)=\bigcup_{s=1}^{T} F_{-i s}
$$

and $p=\bar{p}$ (as defined in Section 2).

- In Game 1, $(O, p)$ describes necessary conditions for investment with

$$
O_{i t}(F)=\bigcup_{s=1}^{t} F_{-i s}
$$

and $p=\frac{c}{b+c}$.

- In Game 2, $(O, p)$ describes sufficient conditions for investment with

$$
O_{i t}(F)=\bigcup_{s=1}^{t+1} F_{-i s}
$$

with the convention that $F_{-i, T+1}=\emptyset$, and $p=\frac{\delta b+c}{b+c}$.

\footnotetext{
${ }^{9}$ In each case, we abuse notation by identifying measurable subsets $F_{i t} \subseteq X_{i t}$ with the ordinary events consisting of all states whose $i t$-coordinate lies in $F_{i t}$.
} 
We restrict attention to sufficient or necessary conditions for investment satisfying four natural properties that are assumed to hold throughout the paper.

Assumption 1. For any $F$ and $F^{\prime}$ such that $F_{-i s}=F_{-i s}^{\prime}$ for all $s, O_{i t}^{T}(F)=O_{i t}^{T}\left(F^{\prime}\right)$ for all $T$ and $(i, t)$.

Assumption 1 states that conditions for investment do not depend on the player's own strategy.

Assumption 2. For each $F$ and $F^{\prime}$ such that $F \subseteq F^{\prime}, O_{i t}^{T}(F) \subseteq O_{i t}^{T}\left(F^{\prime}\right)$ for all $T$ and $(i, t)$.

Assumption 2 states that if the conditions for investment are satisfied, then they continue to be satisfied if the opponent invests on a larger set of types. ${ }^{10}$

Definition 3. Let $\mathcal{D}$ and $\mathcal{R}$ be classes of subsets of partially ordered sets. An operator $H: \mathcal{D} \longrightarrow \mathcal{R}$ is point-monotone if for every upper contour set $D \in \mathcal{D}, H(D)$ is also an upper contour set.

Assumption 3. The operator $O_{i t}^{T}$ is point-monotone for each $T$ and $(i, t)$.

Assumption 3 is a relaxed version of the state monotonicity typically assumed in the global games literature.

The final assumption requires that $O$ satisfy translation and scale invariance, ensuring that conditions for investment are consistent as $\sigma$ varies and across translations of strategy profiles.

Assumption 4. For each $F \in \mathcal{S}, \nu \in(0,1), x \in \mathbf{X}$, and $T, O^{T}(\nu F+x)=\nu O^{T} F+x$.

\subsection{Generalized belief operators}

We characterize rationalizable behavior using a generalization of the common beliefs introduced by Monderer and Samet [13]. In particular, we formulate conditions for rationalizability of investment in terms of generalized common belief that the fundamental is good $(\theta>0)$. To that end, it is useful to introduce a modification of the

\footnotetext{
${ }^{10}$ This assumption is weaker than the usual action monotonicity assumption in static global games that requires the best response correspondence be non-decreasing in the opponent's strategy. Assumption 2 requires monotonicity only of a lower or upper bound of the best response correspondence.
} 
operators $B^{p}$ that captures both beliefs about the fundamental $\theta$ and about the timing of one's opponent's investment.

Given a pair $(O, p)$, define the generalized belief operator $\mathcal{B}_{p}: \Sigma_{\Theta} \cup \mathcal{S} \longrightarrow \mathcal{S}$ by

$$
\mathcal{B}^{p}(F)= \begin{cases}B^{p}(F) & \text { if } F \in \Sigma_{\Theta} \\ B^{p}\left(O^{T} F\right) & \text { if } F \in \mathcal{S} .\end{cases}
$$

Generalized belief $\mathcal{B}^{p}(F)$ is a strategy profile: it is a vector with $2 T$ components, one for each agent $(i, t)$, where each is a set $\mathcal{B}_{i t}^{p}(F)$ of types of agent $(i, t)$. If $F \in \Sigma_{\Theta}$ is an event describing $\theta$ then $\mathcal{B}_{i t}^{p}(F)$ is the set of types of $(i, t)$ that $p$-believe $F$. If $F \in \mathcal{S}$ is a strategy profile then $\mathcal{B}_{i t}^{p}(F)$ is the set of types of $(i, t)$ that, given $F$, $p$-believe that the timing of their opponent's investment satisfies the conditions described by $O{ }^{11}$

The following definition generalizes Monderer and Samet's notion of common beliefs.

Definition 4. For any operator $K: \mathcal{S}^{\prime} \longrightarrow \mathcal{S}$ with $\mathcal{S} \subseteq \mathcal{S}^{\prime}$, let $C_{K}^{1}(F)=K(F)$, and recursively define $C_{K}^{n}$ for $n=2,3, \ldots$ by $C_{K}^{n}(F)=K\left(C_{K}^{n-1}(F)\right) \wedge C_{K}^{n-1}(F)$. The generalized common belief operator $C_{K}$ is defined by

$$
C_{K}(F)=\bigwedge_{n=1}^{\infty} C_{K}^{n}(F)
$$

To intuitively understand the relationship between the generalized common belief operator and Monderer and Samet's notion of common $p$-belief, recall that (standard) common $p$-belief of an event $G$ refers to the event that both players $p$-believe $G$, both $p$-believe that both $p$-believe $G$, and so on. Since the standard notion focuses on fixed beliefs for each player, timing of beliefs is not explicitly considered.

In static environments, generalized common belief corresponds to the standard notion. Suppose that $T=1$ and for each strategy profile $F$, let $O_{i 1}(F)=F_{-i 1}$ be the event that the opponent invests. Then $C_{\mathcal{B}^{p}}$ may be identified with the common $p$-belief operator of Monderer and Samet.

The crucial difference between generalized and standard common beliefs is that generalized common belief allows the timing of beliefs to vary across players and across

\footnotetext{
${ }^{11}$ We suppress the dependence of $\mathcal{B}^{p}$ on $O, T$ and $\sigma$ from the notation, and we abuse notation by identifying $F \in \Sigma_{\Theta}$ with the ordinary event $F \times_{i t} X_{i t}$.
} 
orders of belief in dynamic setups. For example, consider the generalized belief operator with $O_{i t}(F)=\bigcup_{s=1}^{T} F_{-i s}$, which corresponds to our flexible game. Generalized common belief is the event that both players eventually $p$-believe $G$, both eventually $p$-believe that both eventually $p$-believe $G$, and so on. More precisely, generalized common belief $C_{\mathcal{B}^{p}}(G)$ is a vector of length $2 T$ with components $\left[C_{\mathcal{B}^{p}}(G)\right]_{i t}$ where the component indexed by $i t$ is the event that, at period $t$, player $i$-believes

1. $G$, and

2. that there exists $t^{\prime}$ such that $-i p$-believes $G$ at $t^{\prime}$, etc.

The essential difference between this and the hierarchy of beliefs in the standard case is that, at each order, the player is required only to believe that the other player attains the preceding order in some round.

For this particular $O$, the times $t, t^{\prime}$, and those from higher orders are unrelated. This corresponds to the feature of the flexible game that the specific timing of $-i$ 's investment is irrelevant to player $i$. For more restrictive conditions $O$, corresponding to games in which the timing of $-i$ 's investment is relevant to $i$, the timing of beliefs in the above list could be connected.

Next we define an operator, called the expansion operator, that is similar to the generalized common belief operator except with componentwise unions instead of intersections. Our use of the two operators is complementary; we use the generalized common belief operator to identify necessary conditions and the expansion operator to identify sufficient conditions for coordination on the irreversible action.

Definition 5. For any operator $K: \mathcal{S}^{\prime} \longrightarrow \mathcal{S}$ with $\mathcal{S} \subseteq \mathcal{S}^{\prime}$, let $E_{K}^{1}(F)=K(F)$, and recursively define $E_{K}^{n}$ for $n=2,3, \ldots$ by $E_{K}^{n}(F)=K\left(E_{K}^{n-1}(F)\right) \vee E_{K}^{n-1}(F)$. The expansion operator $E_{K}$ is defined by

$$
E_{K}(F)=\bigvee_{n=1}^{\infty} E_{K}^{n}(F) .
$$

\section{Results}

In order to characterize coordination outcomes in terms of generalized higher order beliefs, we first define two concepts describing rationalizable behavior. 
In the inflexible game, players turn out to be "strategically pessimistic" in the sense that they believe that the opponent does not invest unless investing is dominant. In the flexible game, players believe that the opponent invests unless investing is dominated, and are thus "strategically optimistic." The following definitions formalize these notions.

Definition 6. There is strategic pessimism in a class of games $\Gamma$ if for some $\underline{T}$, for each $T>\underline{T}$, there exist $q \in(0,1)$ and $\bar{\sigma}>0$ such that for all $\sigma<\bar{\sigma}$, investing is rationalizable in $\Gamma_{T}(\sigma)$ for type $x_{i t}$ only if

$$
x_{i t} \in B_{i t}^{q}(U) .
$$

Definition 7. There is strategic optimism in a class of games $\Gamma$ if for some $\underline{T}$, for each $T>\underline{T}$, there exist $q \in(0,1)$ and $\bar{\sigma}>0$ such that for all $\sigma<\bar{\sigma}$, investing is the unique rationalizable action in $\Gamma_{T}(\sigma)$ for type $x_{i t}$ whenever

$$
x_{i t} \in B_{i t}^{q}(G) .
$$

Note that in both of the preceding definitions, $q$ may depend on $T$ but not on $\sigma$.

Strategic optimism and pessimism are closely related to the coordination outcome. If there is strategic pessimism, then players fail to coordinate on investing for fundamentals bounded away from the upper dominance region $U$ in games with many rounds and precise signals. Similarly, if there is strategic optimism, then players coordinate on investing without delay for fundamentals bounded away from the lower dominance region $\Theta \backslash G$ in games with many rounds and precise signals.

The next proposition characterizes rationalizability of investment using the generalized common belief operator $C_{\mathcal{B}^{p}}$ and the expansion operator $E_{\mathcal{B}^{p}}$. The first part generalizes the well known necessary condition for rationalizability of investment in static coordination games (see, for example, Morris and Shin 2003). The second part is derived from the first using a duality argument.

Theorem 1. 1. Suppose that $(O, p)$ describes necessary conditions for investment in a class of games $\Gamma$. Suppose moreover that for some $p^{\prime}<p$ and $\underline{T}$, for each $T>\underline{T}$ there exists $\bar{\sigma}>0$ such that $C_{\mathcal{B}^{p^{\prime}}}(G)=\emptyset$ for all $\sigma<\bar{\sigma}$. Then there is strategic pessimism in $\Gamma$. 
2. Suppose that $(O, p)$ describes sufficient conditions for investment in a class of games $\Gamma$. Suppose moreover that for some $p^{\prime}>p$ and $\underline{T}$, for each $T>\underline{T}$ there exists $\bar{\sigma}>0$ such that $E_{\mathcal{B}^{p^{\prime}}}(U)=\mathbf{X}$ for all $\sigma<\bar{\sigma}$. Then there is strategic optimism in $\Gamma$.

The following result describes a condition for strategic optimism that is easier to apply than that of part 2 of Theorem 1.

Theorem 2. Suppose that $(O, p)$ describes sufficient conditions for investment in a class of games $\Gamma$. Suppose moreover that for some $p^{\prime}>p, q \in(0,1)$, and $\underline{T}$, for each $T>\underline{T}$ there exists $\bar{\sigma}$ such that for all $\sigma<\bar{\sigma}$ and all $\theta^{*} \in \mathbb{R}$, we have

$$
B^{q}\left(\theta \geq \theta^{*}\right) \subseteq \mathcal{B}^{p^{\prime}}\left(B^{q}\left(\theta \geq \theta^{*}\right)\right) .
$$

Then there is strategic optimism in $\Gamma$.

The discerning reader may notice that Theorem 2 generalizes the contagion argument from the introductory example. There the event that both players eventually $q$-believe $\theta \geq \theta^{*}$ is evident in a non-simultaneous sense (in sufficiently long games) whenever the event holds each player believes it at some time. As shown in Section 2 , this observation implies the contagion step: if investment is rationalizable whenever players believe $\theta \geq \theta^{*}$ then it is also rationalizable whenever players believe $\theta \geq \theta^{*}-\varepsilon$. Theorem 2 states that if $B^{q}\left(\theta \geq \theta^{*}\right)$ is evident in a generalized sense that reflects sufficient conditions for investment $(O, p)$ then strategic optimism arises. The proof is based on a similar contagion argument.

One can formulate conditions for strategic pessimism symmetric to Theorem 2 based on contagion of not investing from the lower dominance region in games with necessary conditions for investment. We omit such a result because it is not required in the next section where we apply the results of this section to particular games.

\section{Applications}

In this section we apply the preceding theorems to the games introduced in Sections 2 and 3 above. For each game, we show that there is strategic optimism or pessimism. We start with two games that exhibit strategic optimism, followed by two games with strategic pessimism. 


\subsection{The flexible game}

The next proposition essentially replicates Proposition 2 to illustrate the general method of Section 6.

Proposition 3. Suppose that $(O, p)$ describes sufficient conditions for investment in a class of games $\Gamma$, where

$$
O_{i t}^{T}(F)=\bigcup_{s=1}^{T} F_{-i s}
$$

and $p \in(0,1)$. Then there is strategic optimism in $\Gamma$.

Recall that if $p=\bar{p}$ as defined in Section 2, then this pair $(O, p)$ describes sufficient conditions for investment in the flexible game.

Proof. We show that for each $p^{\prime}$ there exist $q$ and $\underline{T}$ such that for each $T>\underline{T}$ and all $\sigma>0$

$$
B^{q}\left(\theta \geq \theta^{*}\right) \subseteq \mathcal{B}^{p^{\prime}}\left(B^{q}\left(\theta \geq \theta^{*}\right)\right)
$$

Theorem 2 then implies strategic optimism in $\Gamma$.

By Lemma 1, we have (for any $(i, t)$ )

$$
\lim _{T \rightarrow \infty} \operatorname{Pr}\left(O_{i t}^{T} B^{q}\left(\theta \geq \theta^{*}\right) \mid \theta^{*}\right)=1 .
$$

Choose $p^{\prime}>p, q>p^{\prime}$, and $\underline{T}$ such that

$$
\operatorname{Pr}\left(O_{i t}^{T} B^{q}\left(\theta \geq \theta^{*}\right) \mid \theta^{*}\right) \geq \frac{p^{\prime}}{q}
$$

for all $T>\underline{T}$ and $\sigma>0$, which is possible since the given probability is independent of $\sigma$. Every type $x_{i t} \in B^{q}\left(\theta \geq \theta^{*}\right)$ assigns probability at least $q \times \frac{p^{\prime}}{q}=p^{\prime}$ to the event $O_{i t}^{T} B^{q}\left(\theta \geq \theta^{*}\right)$, as needed.

\subsection{Game 2}

The following proposition shows that, if learning is sufficiently quick, there is strategic optimism in Game 2 of Section 3. 
Proposition 4. Suppose that $(O, p)$ describes sufficient conditions for investment in a class of games $\Gamma$, where

$$
O_{i t}^{T}(F)=\bigcup_{s=1}^{t+1} F_{-i s},
$$

with the convention that $F_{-i, T+1}=\emptyset$, and $p \in(0,1)$. If there exists $\tau \in(0,1)$ such that $\sigma_{t+1} \leq \tau \sigma_{t}$ for each $t$, then there is strategic optimism in $\Gamma$.

For $p=\frac{\delta b+c}{b+c}$, this pair $(O, p)$ describes sufficient conditions for investment in Game 2 .

Proof. We again show that for each $p^{\prime}$ there exist $q$ and $\underline{T}$ such that for each $T>\underline{T}$ and $\sigma>0$,

$$
B^{q}\left(\theta \geq \theta^{*}\right) \subseteq \mathcal{B}^{p^{\prime}}\left(B^{q}\left(\theta \geq \theta^{*}\right)\right)
$$

Theorem 2 then implies strategic optimism in the class $\Gamma$.

We use the following lemma to prove (6).

Lemma 2. Suppose there exists $\tau \in(0,1)$ such that $\sigma_{t+1} \leq \tau \sigma_{t}$ for every $t$. Then for each $p^{\prime} \in(0,1)$, there exists $q \in(0,1)$ such that

$$
B_{i t}^{q}\left(\theta \geq \theta^{*}\right) \subseteq B_{i t}^{p^{\prime}}\left(B_{-i, t+1}^{q}\left(\theta \geq \theta^{*}\right)\right)
$$

for all $t=1, \ldots, T-1$.

Lemma 2 states that if a player believes $\theta \geq \theta^{*}$, then she also believes that her opponent will believe $\theta \geq \theta^{*}$ in the next period. This property implies that the firstorder belief $B_{q}\left(\theta \geq \theta^{*}\right)$ is evident in a generalized sense reflecting sufficient conditions for investment in this class of games.

Given $p^{\prime}$, let $q$ be as in Lemma 2. The lemma implies that

$$
B_{i t}^{q}\left(\theta \geq \theta^{*}\right) \subseteq B_{i t}^{p^{\prime}}\left(O_{i t}^{T} B^{q}\left(\theta \geq \theta^{*}\right)\right)
$$

for each $t=1, \ldots, T-1$ since $B_{-i, t+1}^{q}\left(\theta \geq \theta^{*}\right) \subseteq O_{i t}^{T} B^{q}\left(\theta \geq \theta^{*}\right)$.

All that remains is to show that in the last round $T$,

$$
B_{i T}^{q}\left(\theta \geq \theta^{*}\right) \subseteq B_{i T}^{p^{\prime}}\left(O_{i T}^{T} B^{q}\left(\theta \geq \theta^{*}\right)\right)
$$


when $T$ is sufficiently large. This last containment follows from the proof of Proposition 3 since $O_{i, T}^{T}=\bigcup_{s=1}^{T} F_{-i s}$ is identical to the corresponding operator for the flexible game.

\subsection{Inflexible game}

The following proposition shows that there is strategic pessimism in the inflexible game of Section 2, in which players invest only in round $T$. The proof essentially replicates the argument for the failure of common knowledge in static global games (see Morris and Shin [15]).

Proposition 5. Suppose that $(O, p)$ describes necessary conditions for investment in a class of games $\Gamma$, where

$$
O_{i t}^{T}(F)= \begin{cases}F_{-i T} & \text { if } t=T \\ \emptyset & \text { otherwise }\end{cases}
$$

and $p>\frac{1}{2}$. Then there is strategic pessimism in $\Gamma$.

Letting $p=\frac{c}{b+c}$, this pair $(O, p)$ describes necessary conditions for investment in the inflexible game.

Proof. Fix $p>\frac{1}{2}$. We show that $C_{\mathcal{B}^{p}}(G)=\emptyset$ for any $T$ and $\sigma>0$. Strategic pessimism then follows from Theorem 1.

Clearly $\left[C_{\mathcal{B}^{p}}(G)\right]_{i t}=\emptyset$ for any $t<T$ by the definition of $O$. Consider round $T$. Recall that $\mathcal{B}_{i T}^{p}(G)$ is the set $\left[\sigma_{T} \Phi^{-1}(p), \infty\right)$ of types of $(i, T)$ that $p$-believe $G$. Turning to the second order of beliefs, $\left[\left(\mathcal{B}^{p}\right)^{2}\right]_{i T}(G)$ is the set of types of $(i, T)$ that $p$-believe that $(-i, T) p$-believes $G$. Conditional on $x_{i T},(i, T)$ believes that $x_{-i T}$ is normally distributed with mean $x_{i T}$ and variance $2 \sigma_{T}^{2}$. Therefore, we have

$$
\left[\left(\mathcal{B}^{p}\right)^{2}(G)\right]_{i T}=\left[\sigma_{T} \Phi^{-1}(p)+\sqrt{2} \sigma_{T} \Phi^{-1}(p), \infty\right)
$$

Proceeding inductively, we see that

$$
\left[C_{\mathcal{B}^{p}}(G)\right]_{i T}=\bigcap_{n}\left[\left(\mathcal{B}^{p}\right)^{n}(G)\right]_{i T}=\bigcap_{n}\left[\sigma_{T} \Phi^{-1}(p)(1+(n-1) \sqrt{2}), \infty\right)=\emptyset
$$


as needed.

\subsection{Game 1}

The following proposition shows that there is strategic pessimism in Game 1 of Section 3.

Proposition 6. Suppose that $(O, p)$ describes necessary conditions for investment in a class of games $\Gamma$, where

$$
O_{i t}^{T}(F)=\bigcup_{s=1}^{t} F_{-i s}
$$

and $p>\frac{1}{2}$. Then there is strategic pessimism in $\Gamma$.

If $p=\frac{c}{b+c}$ then $(O, p)$ describes necessary conditions for investment in Game 1 .

Proof. Fix any $p>\frac{1}{2}$. We show that $C_{\mathcal{B}^{p}}(G)=\emptyset$ for any $T$ and $\sigma$. Strategic pessimism then follows by Theorem 1.

First note that $\left[C_{\mathcal{B}^{p}}(G)\right]_{i 1}$ can be identified with the usual common $p$-belief of $G$, which is empty for $p>\frac{1}{2}$ (as shown in the proof of Proposition 5). The result for periods $t \geq 2$ follows by induction. Suppose $\left[C_{\mathcal{B}^{p}}(G)\right]_{i s}=\emptyset$ for $i=1,2$ and $s=1, \ldots, t-1$. Then $\left[C_{\mathcal{B}^{p}}(G)\right]_{i t}$ coincides with the usual common $p$-belief of $G$ in period $t$, which is again empty.

\section{Discussion}

In this section, we discuss our major assumptions, and consider potential extensions.

In order to simplify the analysis, we restrict the information structure in several ways. For one, we assume that the fundamental is drawn from a uniform prior. As is well understood in the global games literature, this assumption is not important when signals are precise. Any prior with a continuous density leads to posteriors close to those arising from a uniform prior when the noise in signals is small.

A significant feature of our information structure is that players do not observe each other's past choices. Instead, in each period, they observe a noisy private signal $y_{i t}$ of the state $\theta$. The lack of learning from past actions may seem artificial. However, we can reinterpret our model as one with a continuum of players, as described in brief 
below. Under this reinterpretation, in the class of symmetric monotone equilibria, the per-period signals $y_{i t}$ can be shown to be equivalent to noisy observation of the past actions of other players.

To see this, we begin by describing how to reinterpret our flexible game as having a continuum of players of unit measure. Let $l_{t}$ denote the measure of players who have invested by period $t$. Payoffs for investing at $t$ are given by $\delta^{t}\left(b l_{T}-c\left(1-l_{T}\right)\right)$ if $\theta \in(0,1)$, by $b$ if $\theta \geq 1$, and by $-c$ if $\theta \leq 0$. At each time $t$, each player can either invest irreversibly or wait. At $t=1$, let the information structure for each player be identical to that of the original two player game. If players follow symmetric monotone strategies at $t=1$, then there exists an increasing function $\lambda_{1}$ such that $l_{1}=\lambda_{1}(\theta)$. At $t=2$, players observe a monotone statistic based on the measure of first period investors, with some private noise. In particular, suppose that at $t=2$, each player $i$ observes $y_{i 2}=\lambda_{1}^{-1}\left(l_{1}\right)+\widetilde{\sigma}_{2} \varepsilon_{i 2}$, where $\varepsilon_{i 2} \sim N(0,1)$ independently across players. Since $\lambda_{1}^{-1}\left(l_{1}\right)=\theta$, the observation of $y_{i 2}$ is informationally equivalent to the observation of a noisy private signal directly about $\theta$ given by $y_{i 2}=\theta+\widetilde{\sigma}_{2} \varepsilon_{i 2}$. Thus the exogenous private signal at $t=2$ in the original model can be microfounded as a particular form of noisy social learning. But now, if players follow symmetric monotone strategies at $t=2$, there is an increasing function $\lambda_{2}$ such that $l_{2}=\lambda_{2}(\theta)$. In turn, by allowing agents to observe $\lambda_{2}^{-1}\left(l_{2}\right)$ with private noise at $t=3$, we can also reinterpret the private signal at $t=3$ as arising out of the noisy observation of past play. Proceeding iteratively in this way, we can microfound the entire sequence of private signals in the original model. Thus, while our baseline analysis abstracts from the observability of past actions for tractability, our exogenous learning process can be viewed through the lens of endogenous noisy social learning. Although the precise translation between exogenous and social learning relies on particular parametric assumptions, this approach to modeling social learning has become common in the global games literature because of its tractability (see, for example, Angeletos, Hellwig, and Pavan [2] (online supplement), Angeletos and Werning [1], and Goldstein, Ozdenoren, and Yuan [10]).

Another key feature of our examples is that, in each one, one of the two actions is irreversible. However, this feature is not necessary for the general results found in Section 6. Even when actions are reversible in a given game, there may exist a pair $(O, p)$ describing necessary or sufficient conditions for investment based on which we can derive conditions for strategic pessimism or optimism respectively. 


\section{Conclusion}

Static coordination games represent a useful abstraction for studying coordination problems in the real world. However, the associated requirement of simultaneity in participation may be a strong restriction: the outcomes generated in such models may not be good representations of real-world coordination problems where agents are able to participate at different points of time and can learn about payoffs while deciding when to participate. Our results illustrate the radical difference between inflexible and flexible coordination problems within the framework of global games.

This paper introduces a class of global games with enriched timing where agents have many opportunities to participate, while they asymptotically and privately learn the true payoffs. In our benchmark analysis, we consider an extreme version of such games, in which the specific timing of the other player's investment is irrelevant to one's own payoffs. We show that rationalizable play in such a game ensures coordination on the irreversible action, thus differing from the outcome in the inflexible setup.

We also analyze intermediate cases in which players care to some extent about the timing of their opponent's investment. Our results indicate that the coordination outcome can be very sensitive to the precise way in which relative timing of investment influences payoffs. Assuming that investing is irreversible, a small reward for investing before one's opponent results in coordination on investment, as in the benchmark flexible game. A small penalty for investing before one's opponent ensures coordination on the risk-dominant action, as in the canonical static global game. A deeper understanding of timing incentives in dynamic coordination problems may pinpoint details in the design of coordination processes that could help to prevent coordination failures. Our results provide a starting point for such design exercises.

\section{A Appendix}

Proof of Lemma 1. We are indebted to an anonymous referee for suggesting this proof, which is much more transparent than our original proof.

We begin by showing that $l^{q}\left(\theta^{*}, \theta^{*}\right)=1$. To further simplify notation, and without

loss of generality, we consider the case in which $\theta^{*}=0$. In what follows, all distributions are conditional on $\theta=0$. In addition, since the result concerns only the beliefs of a 
single player, we drop the index $i$.

For each $t$, let $H_{t}$ be the event that the player does not $q$-believe $\theta>0$ in period $t$. We need to show that $\lim _{T \rightarrow \infty} \operatorname{Pr}\left(\bigcap_{t=1}^{T} H_{t}\right)=0$. The limit exists because the sequence is decreasing and bounded from below.

We construct an increasing sequence of periods $t_{k}$ such that, for a given $r \in(q, 1)$ and for all $K$,

$$
\operatorname{Pr}\left(H_{t_{K}} \mid \bigcap_{k=1}^{K-1} H_{t_{k}}\right)<r
$$

Then $\operatorname{Pr}\left(\bigcap_{k=1}^{K} H_{t_{k}}\right)<r^{K}$, which converges to 0 as $K \rightarrow \infty$. Since $\operatorname{Pr}\left(\bigcap_{k=1}^{K} H_{t_{k}}\right)>$ $\operatorname{Pr}\left(\bigcap_{t=1}^{t_{K}} H_{t}\right)$, this proves the result.

The construction of the sequence $t_{k}$ is recursive. Let $t_{1}=1$. For $T>t_{K}$,

$$
\operatorname{Pr}\left(H_{T} \mid \bigcap_{k=1}^{K} H_{t_{k}}\right)=\int \operatorname{Pr}\left(H_{T} \mid x_{t_{K}}\right) f\left(x_{t_{K}}\right) d x_{t_{K}}
$$

where $f$ is the distribution of $x_{t_{K}}$ conditional on $\bigcap_{k=1}^{K} H_{t_{k}}$. We show that $\lim _{T \rightarrow \infty} \operatorname{Pr}\left(H_{T} \mid x_{t_{K}}\right)=$ $q$. Therefore, by the Dominated Convergence Theorem, the integral on the right-hand side of (8) converges to $q$. In particular, for $T$ sufficiently large, the integral is less than $r$; choosing some such $T$, let $t_{K+1}=T$.

Let

$$
\frac{1}{\hat{\sigma}_{t}^{2}}=\sum_{t=t_{K}+1}^{T} \frac{1}{\nu_{t}^{2}} \quad \text { and } \quad \hat{x}_{T}=\sum_{t=t_{K}+1}^{T} \frac{\hat{\sigma}_{T}^{2}}{\nu_{t}^{2}} y_{t} .
$$

Thus $\hat{x}_{T}$ captures the information that the player receives in periods $t_{K}+1$ through $T$, and $\frac{1}{\hat{\sigma}_{t}^{2}}$ is the precision of this information. The player's beliefs in period $T$ are determined by the information received by time $t_{K}$ together with the additional information $\hat{x}_{T}$ based on the cumulative signal

$$
x_{t}=\frac{\sigma_{T}^{2}}{\sigma_{t_{K}}^{2}} x_{t_{K}}+\frac{\sigma_{T}^{2}}{\hat{\sigma}_{T}^{2}} \hat{x}_{T}
$$

The player does not $q$-believe $\theta>0$ in period $T$ if and only if $x_{T} / \sigma_{T}<\Phi^{-1}(q)$. 
Hence we have

$$
\operatorname{Pr}\left(H_{T} \mid x_{t_{K}}\right)=\operatorname{Pr}\left(\frac{\sigma_{T}}{\sigma_{t_{K}}^{2}} x_{t_{K}}+\frac{\sigma_{T}}{\hat{\sigma}_{T}^{2}} \hat{x}_{T}<\Phi^{-1}(q)\right) .
$$

As $T \rightarrow \infty$, the term $\frac{\sigma_{T}}{\sigma_{t_{K}}^{2}} x_{t_{K}}$ converges to 0 and $\frac{\sigma_{T}}{\tilde{\sigma}_{T}}$ converges to 1 . Therefore,

$$
\operatorname{Pr}\left(H_{T} \mid x_{t_{K}}\right) \rightarrow \operatorname{Pr}\left(\frac{1}{\hat{\sigma}_{T}} \hat{x}_{T}<\Phi^{-1}(q)\right)=\Phi\left(\Phi^{-1}(q)\right)=q
$$

since $\frac{1}{\hat{\sigma}_{T}} \hat{x}_{T}$ is a standard normal random variable.

To prove that $l^{q}\left(\theta, \theta^{*}\right)$ is continuous in $\theta$ at $\theta^{*}$, note that for each $T, l_{T}^{q}\left(\theta, \theta^{*}\right)$ is continuous in $\theta$ and bounded from above by 1 . Given $\varepsilon>0$, let $T$ be such that $l_{T}^{q}\left(\theta^{*}, \theta^{*}\right)>1-\varepsilon / 2$; such a $T$ exists since $l^{q}\left(\theta^{*}, \theta^{*}\right)=1$. Since $l_{T}^{q}\left(\theta, \theta^{*}\right)$ is continuous, there exists some $\delta>0$ such that $l_{T}^{q}\left(\theta, \theta^{*}\right)>1-\varepsilon$ for every $\theta \in\left(\theta^{*}-\delta, \theta^{*}+\delta\right)$. Since $l_{t}^{q}\left(\theta, \theta^{*}\right)$ is nondecreasing in $t$, it follows that $l^{q}\left(\theta, \theta^{*}\right)>1-\varepsilon$ for every $\theta \in\left(\theta^{*}-\delta, \theta^{*}+\delta\right)$, and therefore $l^{q}\left(\theta, \theta^{*}\right)$ is continuous at $\theta^{*}$ since it is bounded above by 1 .

\section{A.1 Proof of Theorem 1}

Before getting to the main proof we provide two lemmas.

Lemma 3. [Morris and Shin [14] $\left.]^{12}\right]$ Suppose that $K: \mathcal{S} \longrightarrow \mathcal{S}$ is an increasing operator such that for any strategy profile that prescribes action $a \in\{0,1\}$ on $F_{i t}$ for each $(i, t)$, action $a$ is a best response for any agent $(i, t)$ in $\Gamma_{T}(\sigma)$ only on a subset of $K_{i t}(F)$. Then action a is rationalizable in $\Gamma_{T}(\sigma)$ for each agent $(i, t)$ only on some subset of $\left[C_{K}(\mathbf{X})\right]_{i t}$.

Proof. Recall from the definition of generalized common beliefs that $C_{K}^{1}=K(\mathbf{X})$ and $C_{K}^{n}=K\left(C_{K}^{n-1}\right) \wedge C_{K}^{n-1}$ for $n \geq 2$. Consider iterated deletion of never best responses. Action $a$ is never a best response for types outside $K(\mathbf{X})=C_{K}^{1}$ since $K$ is increasing. After one round of deletion, action $a$ is never a best response outside $C_{K}^{2}$. Proceeding in this fashion, action $a$ is deleted for all types of each agent $(i, t)$ outside $\left[C_{K}(\mathbf{X})\right]_{i t}=\bigcap_{n}\left[C_{K}^{n}\right]_{i t}$.

\footnotetext{
${ }^{12}$ There is a small difference between Lemma 3 and Proposition 10 of Morris and Shin [14]. Lemma 3 specifies necessary conditions for rationalizability based on a bound on the best response correspondence whereas Morris and Shin [14] specify necessary and sufficient conditions based directly on the best response correspondence.
} 
If $(O, p)$ describes necessary conditions for investment, then Lemma 3 gives necessary conditions for rationalizability of investment. Define $\tilde{\mathcal{B}}^{p}: \mathcal{S} \longrightarrow \mathcal{S}$ by

$$
\tilde{\mathcal{B}}^{p}(F)=B^{p}\left(\left[G \wedge O^{T} F\right] \vee U\right)
$$

Note that the operator $\tilde{\mathcal{B}}^{p}(F)$ differs from the generalized belief operator $\mathcal{B}^{p}(F)$ defined in Section 5.3, which is independent of $G$ and $U$.

One can express necessary conditions for rationalizability of investment in terms of generalized common belief $C_{\tilde{\mathcal{B}}^{p}}(\mathbf{X})$. However, such a characterization is complicated by the influence of the upper dominance region on the operator $\tilde{B}_{p}$. The following lemma provides a partial characterization of $C_{\tilde{\mathcal{B}}^{p}}(\mathbf{X})$ in terms of the simpler common belief operator $C_{\mathcal{B}^{p}}$, which is independent of the upper dominance region.

First we extend the definition of the operator $\tilde{\mathcal{B}}^{p}$ by replacing the particular sets $G$ and $U$ with arbitrary upper contour sets $Y$ and $Z$ in $\Sigma_{\Theta}$ such that $Y \subseteq Z$. For any $O$ satisfying Assumptions 1 through 4 , define $\tilde{\mathcal{B}}^{p}: \mathcal{S} \longrightarrow \mathcal{S}$ by

$$
\tilde{\mathcal{B}}^{p}(F)=B^{p}\left(\left[Z \wedge O^{T} F\right] \vee Y\right) .
$$

Lemma 4. For each $p^{\prime} \in(0, p)$ and $T$, there exist $q \in(0,1)$ and $\bar{\sigma}>0$ such that

$$
\neg C_{\mathcal{B}^{p^{\prime}}}(Z) \wedge C_{\tilde{\mathcal{B}}^{p}}(\mathbf{X}) \subseteq B^{q}(Y)
$$

for all $\sigma \leq \bar{\sigma}$.

To interpret the statement of the lemma, consider $Z=G$ and $Y=U$. Roughly speaking, the lemma says that if the necessary conditions for rationalizability from Lemma 3 are satisfied in some state where (generalized) common belief of $G$ fails, then players must assign some probability to the upper dominance region $U$. The failure of common belief refers to the common belief operator based on (the simpler) $\mathcal{B}^{p}$, where $\mathcal{B}^{p}$ reflects sufficient conditions for investment $(O, p)$.

Proof of Lemma 4. We prove the equivalent statement

$$
\neg B^{q}(Y) \wedge C_{\tilde{\mathcal{B}}^{p}}(\mathbf{X}) \subseteq C_{\mathcal{B}^{p^{\prime}}}(Z)
$$

Recall that that $O$ is symmetric across players. 
Let $\mathcal{T}(q, \sigma) \subseteq\{1,2\} \times\{1, \ldots, T\}$ be the set of agents $(i, t)$ for which the left hand side of (9) is empty; that is, $\mathcal{T}(q, \sigma)$ is the set of $(i, t)$ for which, given $\sigma$,

$$
\left[C_{\tilde{\mathcal{B}}^{p}}(\mathbf{X})\right]_{i t} \subseteq B_{i t}^{q}(Y)
$$

We claim that $\mathcal{T}(q, \sigma)$ is non-increasing in $q$ and non-decreasing in $\sigma$. The first of these monotonicity properties is trivial. To prove the second, take $\sigma^{\prime}<\sigma$ and suppose $(i, t) \notin \mathcal{T}(q, \sigma)$. We need to show that $(i, t) \notin \mathcal{T}\left(q, \sigma^{\prime}\right)$. Let $\bar{\theta}=\inf Y, \underline{\theta}=\inf Z$, and $R=\left(\frac{\sigma^{\prime}}{\sigma} \underline{\theta}+\left(1-\frac{\sigma^{\prime}}{\sigma}\right) \bar{\theta}, \infty\right)$. Let

$$
\hat{\mathcal{B}}_{\sigma}^{p}(F)=B_{\sigma}^{p}\left(\left[R \wedge O^{T} F\right] \vee Y\right),
$$

where $B_{\sigma}^{p}$ denotes the belief operator when the noise parameter is $\sigma$. Let 1 denote the vector of ones. Letting $\nu=\frac{\sigma^{\prime}}{\sigma}$ and $x=\left(1-\frac{\sigma^{\prime}}{\sigma}\right) \bar{\theta} \mathbf{1}$, for any upper contour set $F$, Assumption 4 implies that

$$
\hat{\mathcal{B}}_{\sigma^{\prime}}^{p}(\nu F+x)=\frac{\sigma^{\prime}}{\sigma} \tilde{\mathcal{B}}_{\sigma}^{p}(F)+\left(1-\frac{\sigma^{\prime}}{\sigma}\right) \bar{\theta} \mathbf{1}
$$

and therefore

$$
C_{\hat{\mathcal{B}}_{\sigma^{\prime}}^{p}}(\mathbf{X})=\frac{\sigma^{\prime}}{\sigma} C_{\tilde{\mathcal{B}}_{\sigma}^{p}}(\mathbf{X})+\left(1-\frac{\sigma^{\prime}}{\sigma}\right) \bar{\theta} \mathbf{1}
$$

Since $\inf \left[C_{\tilde{\mathcal{B}}_{\sigma}^{p}}(\mathbf{X})\right]_{i t} \notin B_{\sigma, i t}^{p}(Y)$ by assumption, (11) implies that $\inf \left[C_{\hat{\mathcal{B}}_{\sigma^{\prime}}^{p}}(\mathbf{X})\right]_{i t} \notin$ $B_{\sigma^{\prime}, i t}^{p}(Y)$. Finally, $C_{\tilde{\mathcal{B}}_{\sigma^{\prime}}^{p}}(\mathbf{X}) \supseteq C_{\hat{\mathcal{B}}_{\sigma^{\prime}}^{p}}(\mathbf{X})$ since $Z \supseteq R$, and therefore $\inf \left[C_{\tilde{\mathcal{B}}_{\sigma^{\prime}}^{p}}(\mathbf{X})\right]_{i t} \notin$ $B_{\sigma^{\prime}, i t}^{p}(Y)$, as needed.

Let

$$
\begin{aligned}
\mathcal{T}(q) & =\bigcap_{\sigma>0} \mathcal{T}(q, \sigma) \\
\text { and } \quad \mathcal{T} & =\bigcup_{q \in(0,1)} \mathcal{T}(q) .
\end{aligned}
$$

Note that the lemma holds for all components $(i, t) \in \mathcal{T}$ : there exists $q$ such that all $(i, t) \in \mathcal{T}$ belong to the set $\mathcal{T}(q, \sigma)$ for sufficiently small $\sigma$, and (9) holds trivially for such $(i, t)$ because the left-hand side is empty.

It remains to show that the lemma holds for all $(i, t) \notin \mathcal{T}$. Take any such $(i, t)$. 
We have $(i, t) \notin \mathcal{T}(q)$ for each $q>0$, and hence, by the monotonicity of $\mathcal{T}(q, \sigma)$ with respect to $\sigma$, for each $q>0$ there exists $\bar{\sigma}$ such that $(i, t) \notin \mathcal{T}(q, \sigma)$ for each $\sigma \leq \bar{\sigma}$.

Let $q^{\prime}$ and $\bar{\sigma}^{\prime}$ be such that $\mathcal{T} \subseteq \mathcal{T}\left(q^{\prime}, \sigma\right)$ for all $\sigma \leq \bar{\sigma}^{\prime}$; such $q^{\prime}$ and $\bar{\sigma}^{\prime}$ exist because $\mathcal{T}$ is finite. Let $q<q^{\prime}$ and $\bar{\sigma}^{\prime \prime}>0$ be such that $\mathcal{T}(q, \sigma) \subseteq \mathcal{T}$ for all $\sigma \leq \bar{\sigma}^{\prime \prime}$. Letting $\bar{\sigma}=\min \left\{\bar{\sigma}^{\prime}, \bar{\sigma}^{\prime \prime}\right\}$, we have $\mathcal{T}=\mathcal{T}(q, \sigma)$ for all $\sigma \leq \bar{\sigma}$ since $\mathcal{T}(q, \sigma)$ is non-increasing in $q$. Henceforth, we restrict $\sigma$ to be at most $\bar{\sigma}$.

Let $\tilde{C}^{n}=C_{\tilde{\mathcal{B}}^{p}}^{n}(\mathbf{X})$ and $C^{n}=C_{\mathcal{B}^{p^{\prime}}}^{n}(Z)$, and similarly for $\tilde{C}$ and $C$. Let $x_{i t}^{*}=\inf \tilde{C}_{i t}$. For each $(i, t) \notin \mathcal{T}$, we have $x_{i t}^{*} \notin B_{i t}^{q}(Y)$, for otherwise (10) would hold for $(i, t)$ and $(i, t)$ would belong to $\mathcal{T}(q, \sigma)=\mathcal{T}$.

We prove by induction that $\tilde{C}_{i t}^{n} \subseteq C_{i t}^{n}$ for all $n$ and all $(i, t) \notin \mathcal{T}$. This implies that $\tilde{C}_{i, t} \subseteq C_{i t}$ for all $t \notin \mathcal{T}$, as needed.

The statement $\tilde{C}_{i t}^{n} \subseteq C_{i t}^{n}$ holds trivially for $n=1$. Assume for induction that the statement holds for $n-1$ for all $(i, t) \notin \mathcal{T}$. We show that $\tilde{C}_{i t}^{n} \subseteq C_{i t}^{n}$, that is, that

$$
B_{i t}^{p}\left(\left(Z \cap O_{i t} \tilde{C}^{n-1}\right) \cup Y\right) \cap \tilde{C}_{i t}^{n-1} \subseteq B_{i t}^{p^{\prime}}\left(O_{i t} C^{n-1}\right) \cap C_{i t}^{n-1}
$$

Let $x_{i t}^{n}=\inf \tilde{C}_{i t}^{n}$. Note that, by induction, $\tilde{C}^{n}$ is a closed upper contour set for each $n$ since $B_{p}(S)$ is closed for any upper contour set $S$, and hence $x_{i t}^{n} \in \tilde{C}_{i t}^{n}$. Since $x_{i t}^{n} \leq x_{i t}^{*}$, it follows that $x_{i t}^{n} \notin B_{i t}^{q}(Y)$.

For $q$ sufficiently small relative to $q^{\prime}$, the type $x_{i t}^{n}$ assigns arbitrarily small probability to the event $Y \bigcup_{t:(-i, t) \in \mathcal{T}} B_{-i t}^{q^{\prime}}(Y)$, independent of $\sigma$. Hence, for sufficiently small $q$, $x_{i t}^{n} \in B_{i t}^{p}\left(\left(Z \cap O_{i t} \tilde{C}^{n-1}\right) \cup Y\right)$ implies that

$$
x_{i t}^{n} \in B_{i t}^{p^{\prime}}\left(Z \cap \hat{O}_{i t} \tilde{C}^{n-1}\right),
$$

where the operator $\hat{O}_{i t}$ is defined by

$$
\hat{O}_{i t}(F)=O_{i t}(Q(F)) \text {, }
$$

where

$$
Q(F)_{i t}= \begin{cases}\emptyset & \text { if }(i, t) \in \mathcal{T} \\ F_{i t} & \text { otherwise. }\end{cases}
$$


Next, we have

$$
x_{i t}^{n} \in B_{i t}^{p^{\prime}}\left(Z \cap \hat{O}_{i t} \tilde{C}^{n-1}\right) \subseteq B_{i t}^{p^{\prime}}\left(\hat{O}_{i t} \tilde{C}^{n-1}\right) \subseteq B_{i t}^{p^{\prime}}\left(\hat{O}_{i t} C^{n-1}\right),
$$

where the last containment follows from the induction hypothesis that $\left[\tilde{C}^{n-1}\right]_{i t} \subseteq$ $\left[C^{n-1}\right]_{i t}$ for all $(i, t) \notin \mathcal{T}$, together with $\hat{O}$ being increasing. Finally, we have

$$
x_{i t}^{n} \in B_{i t}^{p^{\prime}}\left(\hat{O}_{i t} C^{n-1}\right) \subseteq B_{i t}^{p^{\prime}}\left(O_{i t} C^{n-1}\right)
$$

because $\hat{O}_{i t} F \subseteq O^{i t} F$ for any $F$. Since $x_{i t}^{n} \in \tilde{C}_{i t}^{n-1} \subseteq C_{i t}^{n-1}$ for all $(i, t) \notin \mathcal{T}$, we have $x_{i t}^{n} \in B_{i t}^{p^{\prime}}\left(O_{i t} C^{n-1}\right) \cap C_{i t}^{n-1}$ for all $(i, t) \notin \mathcal{T}$.

All that remains is to show that $B^{p^{\prime}}\left(O C^{n-1}\right) \wedge C^{n-1}$ is an upper contour set. This follows by induction on $n$ since $O$ and $B^{p^{\prime}}$ are point-monotone and $A \wedge B$ is an upper contour set whenever $A$ and $B$ are.

Proof of Theorem 1. First we prove part 1. By Lemma 3, action 1 is rationalizable in $\Gamma_{T}(\sigma)$ only on a subset of $C_{\tilde{\mathcal{B}}^{p}}(\mathbf{X})$, where the operator $\tilde{\mathcal{B}}^{p}: \mathcal{S} \longrightarrow \mathcal{S}$ is defined by

$$
\tilde{\mathcal{B}}^{p}(F)=B^{p}([G \wedge O F] \vee U) .
$$

Suppose that for some $r \in(0, p), C_{\mathcal{B}^{p-r}}(G)=\emptyset$ whenever $T$ is sufficiently large and $\sigma \leq \bar{\sigma}$ (given $T$ ). By Lemma 4 with $Y=U$ and $Z=G$, we have $C_{\tilde{\mathcal{B}}^{p}}(\mathbf{X}) \subseteq B^{q}(U)$ for some $q \in(0,1)$. Therefore, action 1 is rationalizable in $\Gamma_{T}(\sigma)$ (for all sufficiently large $T$ and $\sigma \leq \bar{\sigma})$ only if agent $(i, t) q$-believes $U$, as claimed.

Next we prove part 2. Suppose $(O, p)$ describes sufficient conditions for investment in a class of games $\Gamma$. Consider a strategy profile $F$ in a game $\Gamma_{T}(\sigma)$. Action 0 is a best response for type $x_{i t}$ in $\Gamma_{T}(\sigma)$ only if

$$
x_{i t} \notin B_{i t}^{p}\left(\left[G \cap O_{i t} F\right] \cup U\right),
$$


or equivalently, only if

$$
\begin{aligned}
x_{i t} & \in B_{i t}^{1-p}\left[\overline{\left(\left[G \cap O_{i t} F\right] \cup U\right)}\right] \\
& =B_{i t}^{1-p}\left(\left[\bar{G} \cup \overline{O_{i t} F}\right] \cap \bar{U}\right) \\
& =B_{i t}^{1-p}\left(\left[\bar{U} \cap \overline{O_{i t} F}\right] \cup \bar{G}\right),
\end{aligned}
$$

where $\bar{X}$ denotes the complement of an event $X$.

By Lemma 3, action 0 is rationalizable in $\Gamma_{T}(\sigma)$ only on $C_{\hat{\mathcal{B}}^{1-p}}(\mathbf{X})$, where the operator $\hat{\mathcal{B}}^{1-p}: \mathcal{S} \longrightarrow \mathcal{S}$ is defined by

$$
\hat{\mathcal{B}}^{1-p}(F)=B^{1-p}([\bar{U} \wedge \neg O F] \vee \bar{G})
$$

where $\neg O$ is defined component-wise by $\neg O_{i t}^{T}(F)=\overline{O_{i t}^{T}(F)}$. Note that since $\neg O$ is decreasing, the operator $\hat{\mathcal{B}}^{1-p}$ satisfies the monotonicity requirement for $K$ in Lemma 3: if $F^{\prime}$ prescribes action 0 to more types than does $F$, then $\hat{\mathcal{B}}^{1-p}\left(F^{\prime}\right)$ prescribes 0 to more types than does $\hat{\mathcal{B}}^{1-p}(F)$.

Applying Lemma 4 with $Y=\bar{G}$ and $Z=\bar{U}$, we find that for each $r \in(0,1-p)$,

$$
\left(\neg C_{\mathcal{B}^{1-p-r}}(\bar{U}) \wedge C_{\hat{\mathcal{B}}^{1-p}}(\mathbf{X})\right) \subseteq B^{q}(\bar{G})
$$

for some $q \in(0,1)$.

Suppose that for some $r \in(0,1-p)$,

$$
\neg C_{\mathcal{B}^{1-p-r}}(\bar{U})=\mathbf{X}
$$

whenever $T$ is sufficiently large and $\sigma \leq \bar{\sigma}$. Then (12) implies that

$$
C_{\hat{\mathcal{B}}^{1-p}}(\mathbf{X}) \subseteq B^{q}(\bar{G})
$$

which in turn implies that $\neg B^{q}(\bar{G}) \subseteq \neg C_{\hat{\mathcal{B}}^{1-p}}(\mathbf{X})$, and therefore

$$
B^{1-q}(G) \subseteq \neg C_{\hat{\mathcal{B}}^{1-p}}(\mathbf{X})
$$

Thus, given (13), if $(i, t)(1-q)$-believes $G$ then not investing is not rationalizable and hence investing is the unique rationalizable action (for sufficiently large $T$ and $\sigma \leq \bar{\sigma}$ ). 
It remains to show that (13) is equivalent to

$$
E_{\mathcal{B}^{p+r}}(U)=\mathbf{X}
$$

This equivalence follows from the following duality argument. We say that $L$ is the dual operator to $K$ if $L(F)=\neg K(\neg F)$ for all $F$. One may readily verify that if $L$ is dual to $K$ then $E_{L}$ is dual to $C_{K}$. Noting that $\mathcal{B}^{1-p-r}(F)=\neg \mathcal{B}^{p+r}(\neg F)$, the equivalence follows from the fact that $\mathcal{B}^{1-p-r}$ is dual to $\mathcal{B}^{p+r}$.

\section{A.2 Additional Proofs}

Proof of Theorem 2. We prove that (3) implies that there exists $r>p$ such that $E_{\mathcal{B}^{r}}(U)=\mathbf{X}$ whenever $T$ is sufficiently large and $\sigma \leq \bar{\sigma}$ (given $T$ ). This result in turn implies strategic optimism by part 2 of Theorem 1.

Define the function $\pi_{i t}: X_{i t} \longrightarrow[0,1]$ by

$$
\pi_{i t}(x)=\operatorname{Pr}\left(O_{i t}^{T} B^{q}\left(\theta \geq \theta^{*}\right) \mid x_{i t}=x\right) .
$$

Note that the dependence of $\pi_{i t}$ on $\sigma, T$, and $\theta^{*}$ is suppressed from the notation. The function $\pi_{i t}(\cdot)$ is continuous since posterior beliefs are continuous in types.

Relation (3) implies that $\pi_{i t}(x) \geq p^{\prime}$ for all $x \geq \theta^{*}+\sigma_{t} \Phi^{-1}(q)$. Choose some $r \in\left(p, p^{\prime}\right)$. By the continuity of $\pi_{i t}$, there exists $\varepsilon_{i t}>0$ such that $\pi_{i t}(x) \geq r$ for all $x \geq \theta^{*}+\sigma_{t} \Phi^{-1}(q)-\varepsilon_{i t}$. Thus we have

$$
B_{i t}^{q}\left(\theta \geq \theta^{*}-\varepsilon_{i t}\right) \subseteq \mathcal{B}_{i t}^{r}\left(B^{q}\left(\theta \geq \theta^{*}\right)\right)
$$

Letting $\varepsilon=\min _{(i, t)} \varepsilon_{i t}$, it follows that

$$
B^{q}\left(\theta \geq \theta^{*}-\varepsilon\right) \subseteq \mathcal{B}^{r}\left(B^{q}\left(\theta \geq \theta^{*}\right)\right)
$$

where $\varepsilon$ depends on $T$ and $\sigma$ but is always strictly positive.

Let $E^{1}=B^{r}(U)$, and recursively define $E^{k}=\mathcal{B}^{r}\left(E^{k-1}\right) \vee E^{k-1}$ for $k=2,3 \ldots$

We prove that

$$
B^{q}(\theta \geq 1-k \varepsilon) \subseteq E^{k+1}
$$


for each $k$. For $q>r$, the statement holds trivially for $k=0$. Suppose for induction that the statement holds for $k-1$. Then

$$
B^{q}(\theta \geq 1-(k-1) \varepsilon-\varepsilon) \subseteq \mathcal{B}^{r}\left(B^{q}(\theta \geq 1-(k-1) \varepsilon)\right) \subseteq \mathcal{B}^{r}\left(E^{k}\right) \subseteq E^{k+1}
$$

where the first containment follows from (14) with $\theta^{*}=1-(k-1) \varepsilon$, and the second from the fact that $\mathcal{B}^{r}$ is increasing.

By (15), we have

$$
\bigvee_{k=0}^{\infty} B^{q}(\theta \geq 1-k \varepsilon) \subseteq \bigvee_{k=0}^{\infty} E^{k+1}=E_{\mathcal{B}^{r}}(U)
$$

Since $\bigvee_{k=0}^{\infty} B^{q}(\theta \geq 1-k \varepsilon)=\mathbf{X}$, it follows that $E_{\mathcal{B}^{r}}(U)=\mathbf{X}$, as needed.

Proof of Lemma 2. The set $B_{-i, t+1}^{q}\left(\theta \geq \theta^{*}\right)$ consists of those $x_{-i, t+1}$ exceeding $\theta^{*}+$ $\sigma_{t+1} \Phi^{-1}(q)$ and $x_{-i, t+1} \mid x_{i t} \sim N\left(x_{i t}, \sigma_{t}^{2}+\sigma_{t+1}^{2}\right)$. Therefore

$$
\operatorname{Pr}\left(B_{-i, t+1}^{q}\left(\theta \geq \theta^{*}\right) \mid x_{i t}\right)=\Phi\left(\frac{x_{i t}-\theta^{*}-\sigma_{t+1} \Phi^{-1}(q)}{\sqrt{\sigma_{t}^{2}+\sigma_{t+1}^{2}}}\right) .
$$

The set $B_{i t}^{q}\left(\theta \geq \theta^{*}\right)$ consists of all $x_{i t}$ exceeding $\theta^{*}+\sigma_{t} \Phi^{-1}(q)$. Thus for every $x_{i t} \in B_{i t}^{q}\left(\theta \geq \theta^{*}\right)$, the right-hand side of (16) is at least

$$
\Phi\left(\frac{\theta^{*}+\sigma_{t} \Phi^{-1}(q)-\theta^{*}-\sigma_{t+1} \Phi^{-1}(q)}{\sqrt{\sigma_{t}^{2}+\sigma_{t+1}^{2}}}\right)=\Phi\left(\Phi^{-1}(q) \frac{1-\tau_{t}}{\sqrt{1+\tau_{t}^{2}}}\right)
$$

where $\tau_{t}=\sigma_{t+1} / \sigma_{t}$. The last expression is decreasing in $\tau_{t}$ for $\tau_{t} \in(0,1)$. Since $0<\tau_{t} \leq \tau<1$ by assumption, each type $x_{i t} \in B_{i t}^{q}\left(\theta \geq \theta^{*}\right)$ assigns probability at least $\Phi\left(\Phi^{-1}(q) \frac{1-\tau}{\sqrt{1+\tau^{2}}}\right)$ to $B_{-i, t+1}^{q}\left(\theta \geq \theta^{*}\right)$. For $q$ sufficiently large, $\Phi\left(\Phi^{-1}(q) \frac{1-\tau}{\sqrt{1+\tau^{2}}}\right) \geq p^{\prime}$, as needed.

\section{References}

[1] G.M. Angeletos and I. Werning. Crises and prices: Information aggregation, multiplicity, and volatility. The American economic review, 96(5):1720-1736, 2006. 
[2] G.M. Angeletos, C. Hellwig, and A. Pavan. Dynamic global games of regime change: Learning, multiplicity, and the timing of attacks. Econometrica, 75(3): 711-756, 2007.

[3] H. Carlsson and E. Van Damme. Global games and equilibrium selection. Econometrica: Journal of the Econometric Society, 61(5):989-1018, 1993.

[4] C. Chamley. Dynamic speculative attacks. The American Economic Review, 93 (3):603-621, 2003.

[5] M.W. Cripps, J.C. Ely, G.J. Mailath, and L. Samuelson. Common learning. Econometrica, 76(4):909-933, 2008.

[6] A. Dasgupta. Coordination and delay in global games. Journal of Economic Theory, 134(1):195-225, 2007.

[7] A.K. Dixit and R.S. Pindyck. Investment under uncertainty. Princeton University Press Princeton, NJ, 1994.

[8] J. Ely. Common learning. Mimeo, 2003.

[9] D. Gale. Dynamic coordination games. Economic theory, 5(1):1-18, 1995.

[10] I. Goldstein, E. Ozdenoren, and K. Yuan. Learning and Complementarities in Speculative Attacks. Mimeo, 2009.

[11] P. Heidhues and N. Melissas. Equilibria in a dynamic global game: the role of cohort effects. Economic Theory, 28(3):531-557, 2006.

[12] J. Horner. Payoff-Dominance in Dynamic Coordination Games. 2004.

[13] D. Monderer and D. Samet. Approximating common knowledge with common beliefs. Games and Economic Behavior, 1(2):170-190, 1989.

[14] S. Morris and H.S. Shin. Common Belief Foundations of Global Games. Mimeo.

[15] S. Morris and H.S. Shin. Global games: theory and applications. In Advances in Economics and Econometrics: theory and applications, Eighth world Congress, volume 1, pages 56-114, 2003. 
[16] D.G. Pearce. Rationalizable strategic behavior and the problem of perfection. Econometrica: Journal of the Econometric Society, 52(4):1029-1050, 1984.

[17] J. Xue. Endogenous Timing and Efficiency in Coordination Games with Incomplete Information. Mimeo, 2003. 